\title{
Implementation of Wildlife Animals Detection and Protection System using Raspberry Pi
}

\author{
K. S. Murugesan, AP (Sl.Gr.) ${ }^{1}$, R. Gopalan, AP (Sl.Gr.) $)^{2}$, T. Sathya, AP (Sr.Gr.) ${ }^{3}$, \\ G. Boopathi Raja, AP (Sr.Gr.) ${ }^{4}$
}

Department of Electronics \& Communication Engineering, Velalar College of Engineering \& Technology, Erode

\begin{abstract}
This paper is focused on the protection of crops from the wild animals which often damages the crops by self- feeding or simply by running over the field and crushing the crops. In this paper, Raspberry Pi is used to detect and track the motion of the animals. Using image processing techniques the animal is detected precisely and a tranquilizer gun is used with sedatives to control the animals. Also the information would be passed on to the nearest forest officers and villagers selectively, thereby reducing the crop damages and also efficiently conserve the wildlife which often gets affected from the existing methods like electric fences or other human weapons.
\end{abstract}

Keywords: Raspberry Pi, Wild Animals, Image Processing, Tranquilizer Gun

\section{INTRODUCTION}

In recent years, the protection of crops from the wild animals are very challenging factor. Animals such as elephants, wild boars, moles, monkeys and many others which may often damage the crops by self-feeding or simply by running over the fields. Also there are instances where human lives are lost due to the wild animals attack. The existing methods like electric fences, creating unpleasant noise and detecting the animals using RFID and LF tags which are injected into the animal's skin are proving to be less efficient, as few methods compromise on the safety of wild life and few on the human lives. In this paper, the motion detection and tracking of animals can be controlled by Raspberry Pi system. Using image processing techniques, the animal is detected precisely and a tranquilizer gun is used with sedatives to control the animal. Also the information would be passed on to the nearest forest officers thereby reducing the crop damages and minimizing the probable loss to the farmers.

\section{LITERATURE SURVEY}

Divya, Usha Krishnan et al, proposed the method to use electric fences to protect the crops from the wild animals. But by using this method, there are chances of animals getting hurt due to high electricity. And it is not only the wild animals, but also the pet animals and human beings might also be affected dangerously.

S Santhiya, M Surekha et al, proposed the method to study the behavior of the animal and create different sounds that irritate the particular animal. The animal can be detected using RFID and LF tags which are injected into the animal's skin. This method can be dangerous to the wild animals since the injected tags can create infection. Also it is highly impossible to inject RFID and LF tags to all the wild animals in any forest area.

\section{PROPOSED METHODS}

Using this paper, the motion of the animal can be precisely detected and tracked using image processing techniques and a tranquilizer gun is used with sedatives to control the animal. Also the information would be passed on to the nearest forest officers thereby reducing the crop damages and minimizing the probable loss to the farmers. The block diagram of wildlife animals detection and protection system using Raspberry Pi with required controls are shown in Figure 1.

The image is captured by the USB camera. The camera capture the images in a frame by frame manner, several frames which are being received are to be compared. The captured image thus gets processed. This enables live monitoring. The processing is carried out using OPENCV TOOL. The OPENCV TOOL has its applications extended from python script. The image obtained is being compared with the relative one in order to carry out the comparison an algorithm has been used. The motion detection algorithm is used. The stipulated nature of the algorithm is to compare the frames obtained and to detect the motion relatively. Thus by comparing the relative frames the motion of objects could be determined. This technique is being implemented to detect the motion of animals in forestry. This processing is carried out using Raspberry $\mathrm{Pi}$, which could compare the frames relatively and transmit the information. 


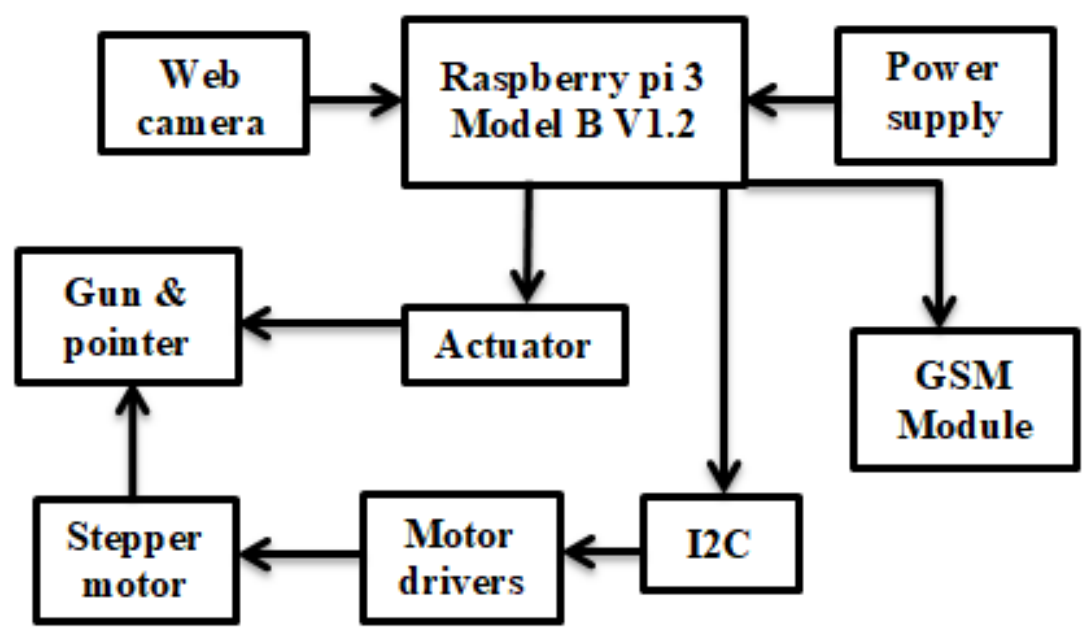

Figure 1 Block diagram

The information is being transmitted via UART, based on the results of the algorithm. The information is being transferred to the GSM module. The GSM module could intimate the forest officer regarding the animal's entry into a particular residential zone. This could also be used intimate if a person's entry into buffer zone of the forest.

The information which is received will be monitored by the forest officer. If the officer wants to have a look at the particular area and detect the intruding animal he can monitor it by accessing the Raspberry Pi. The Raspberry Pi is accessed by Virtual Network Computing (VNC). Virtual Networking Computing is utilized to control the Raspberry Pi whereas its actual function is to monitor the performance of one system module in another system module. In case of detection of any animal the officer could activate the tranquilizer gun. The tranquilizer could be used to shot at the particular animal. The dosage could be varied based on the animal being detected. The animal could be aimed by using tranquilizer guns incorporated with aiming to around 360 degree horizontally and 180 degree vertically.

\section{A. RaspberryPi}

The Raspberry Pi is shown in Figure 2. It is a series of small single board computers to promote teaching of the basic computer science. Several generations of the Raspberry Pi's have been released. All models feature a Broadcom System on a Chip (SoC) with an integrated ARM - Compatible Central Processing Unit (CPU) and on-chip Graphics Processing Unit (GPU).

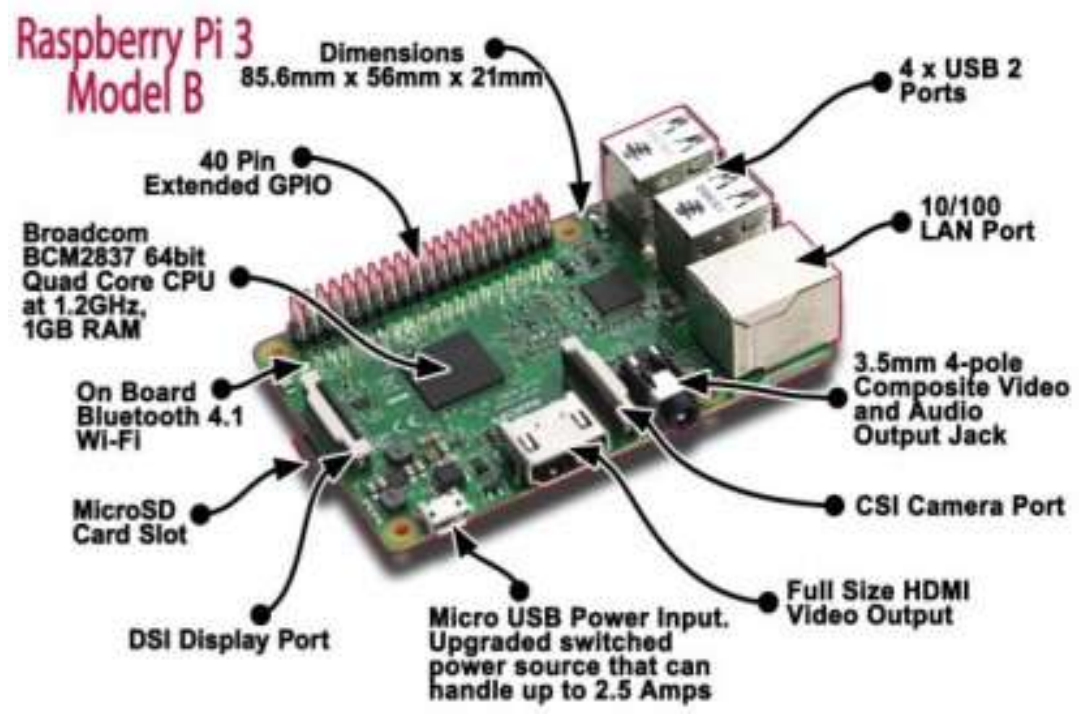

Figure 2 Raspberry Pi

B. ImageProcessing

The images captured with a real-time camera are first scanned thoroughly and compared with the reference images already stored in the system. After comparing the captured images, the system evaluates the result to determine whether the detected subject is an animal or something else. Figure 3 shows the motion detection. 
Vol. 8, Issue 3, March 2019

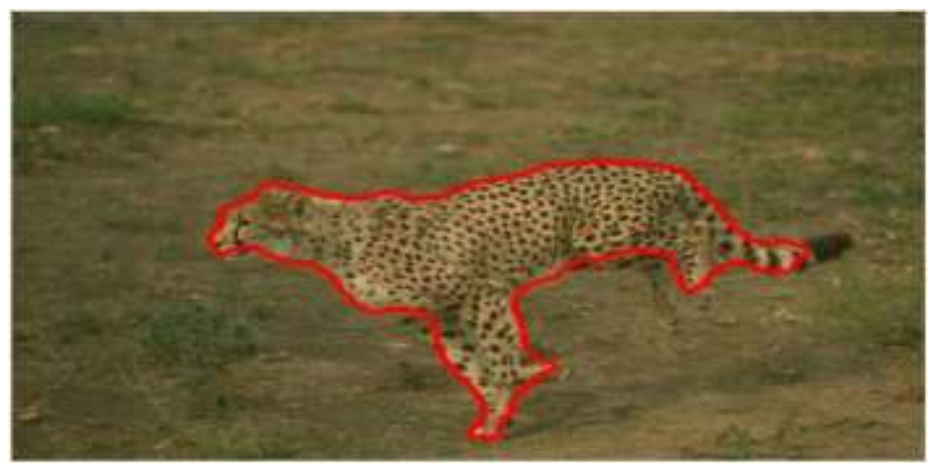

Figure 3 Motion detection

\section{Stepper MotorHat}

It can drive two stepper motors with full PWM speed control. It has fully-dedicated PWM driver chip on board to both control motor direction and speed. This chip can handle all motors and speed controls over $\mathrm{I}^{2} \mathrm{C}$. Motors are controlled by TB6612 drivers. Polarity protected two pin terminal block and jumper to connect external 5-12 DC power. Stepper Motor Hat shown in Figure 4.

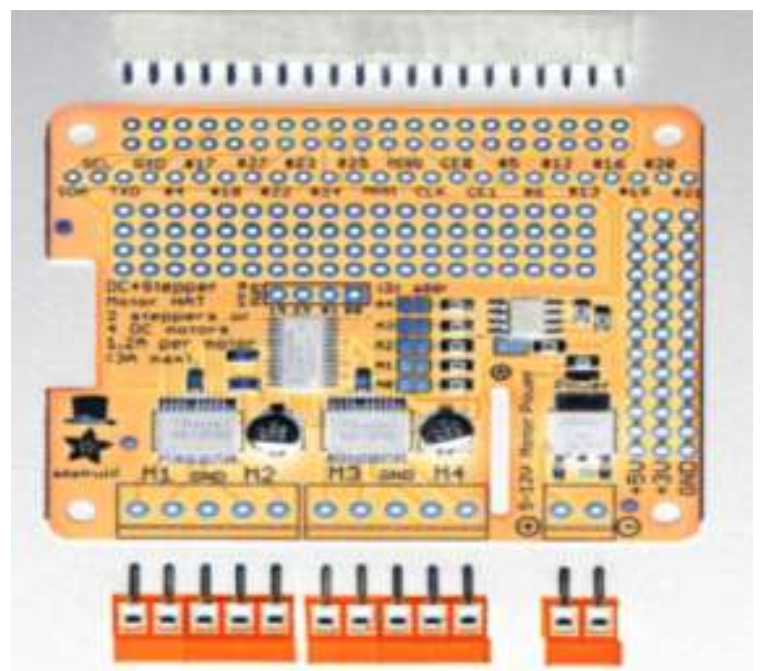

Figure 4 Stepper MotorHat

\section{Stepper Motor}

A stepper motor is four phase unipolar permanent magnet. It is standard size is 200 steps per revolution $12 \mathrm{~V}$ motor. Each step will turn the shaft only 1.8 degree for a full step or 0.9 degree in half stepping motor shown in Figure 5 .

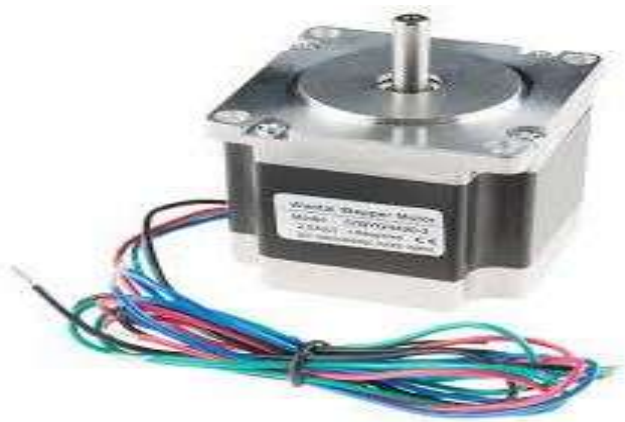

Figure 5 Stepper motor

\section{E. Web Camera}

A webcam is a video camera that feeds or streams its image in real time to or through a computer to a computer network. When "captured" by the computer, the video stream may be saved, viewed or sent on to other networks travelling through systems such as the internet, and e-mailed as an attachment. When sent to a remote location, the video stream may be saved, viewed or on sent there. Unlike an IP camera (which connects using Ethernet or Wi-Fi), a 
webcam is generally connected by a USB cable, or similar cable, are built into computer hardware, such as laptops. Logitech-C170 webcam is used and show in Figure 6. Web-cams typically include a lens, an image sensor, support electronics, and may also include a microphone for sound.

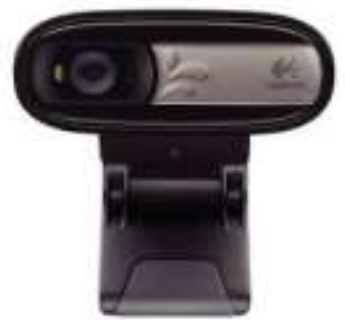

Figure 6 Logitech-C170 Webcam

Various lenses are available, the most common in consumer-grade web-cams being a plastic lens that can be screwed in and out to focus the camera. Most consumer web-cams are capable of providing VGA-resolution video at a frame rate of 30 frames per second. Many newer devices can produce video in multi-megapixel resolutions and a few can run at high frame rates such as the Play Station eye, which can produce 320x240 video at 120 frames per second.

\section{F. GSM Module}

A GSM module is a specialized type of modem which accepts a SIM card, and operates over a subscription to a mobile operator, just like a mobile phone. The mobile operator perspective, a GSM module is shown in Figure 7.

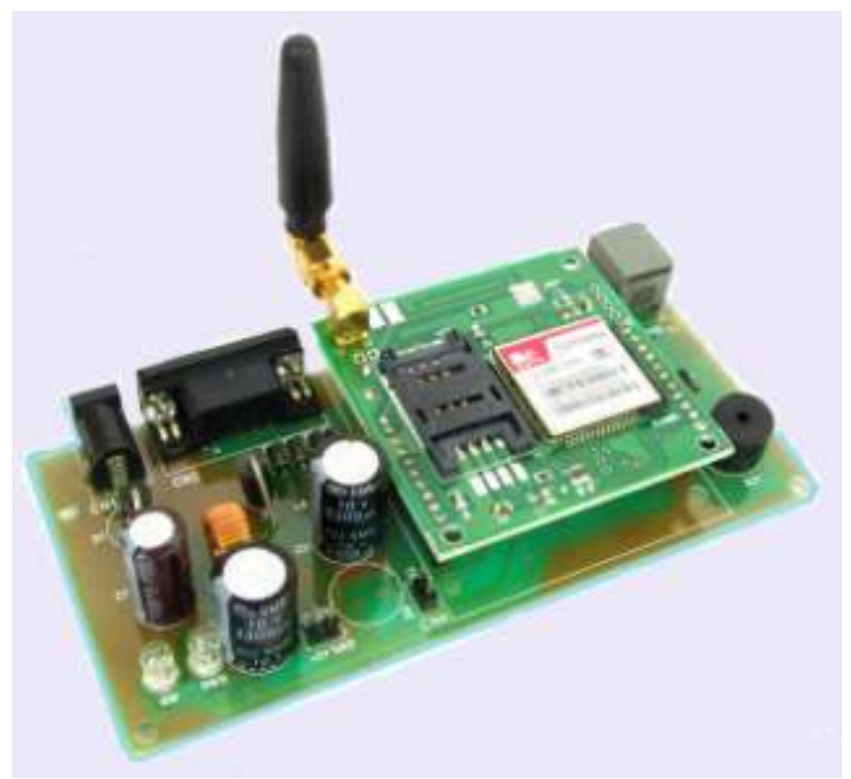

Figure 7 GSM module

When a GSM module is connected to a computer, this allows the computer to use the GSM module to communicate over the mobile network. While these GSM modules are most frequently used to provide mobile internet connectivity, many of them can also be used for sending and receiving SMS and MMS messages. GSM networks operate in a number of different carrier frequency ranges (separated into GSM frequency ranges for 2G and UMTS frequency bands for 3G), with most 2G GSM networks operating in the $900 \mathrm{MHz}$ or $1800 \mathrm{MHz}$ bands.

\section{G. $\quad \mathrm{I}^{2} \mathrm{C}$ Interfacing}

$\mathrm{I}^{2} \mathrm{C}$ is a serial communication protocol. Data is transferred bit by bit along a single wire (the SDA line). $\mathrm{I}^{2} \mathrm{C}$ is synchronous, so the output of bits is synchronized to the sampling of bits by a clock signal shared between the master and the slave. The clock signal is always controlled by the master.

The communication signal of $\mathrm{I}^{2} \mathrm{C}$ is shown in Figure 8. SDA (Serial Data) - The line for the master and slave to send and receive data. SCL (Serial Clock) - The line that carries the clock signal. $\mathrm{I}^{2} \mathrm{C}$ is appropriate for peripherals where simplicity and low manufacturing cost are more important than speed. Common applications of the $\mathrm{I}^{2} \mathrm{C}$ bus are:

1) Describing connectable devices via small ROM configuration tables to enable "plug and play" operation. 
2) System management for PC systems via SMBus.

3) Accessing real-time clocks and NVRAM chips that keep user settings.

4) Accessing low-speed DACs and ADCs.

5) Changing contrast, hue, and color balance settings in monitors.

6) Changing sound volume in intelligent speakers.

7) Reading hardware monitors and diagnostic sensors, e.g. a fan's speed.

8) Turning on and turning off the power supply of system components.

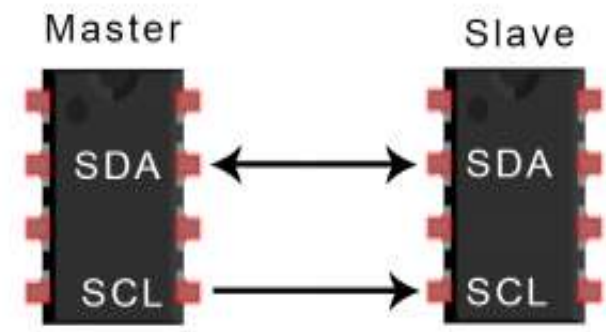

Figure 8 I2C Communication

H. Tranquilizer Gun

A tranquilizer gun used for incapacitating animal targets via drugs usually referred as tranquilizers. These gun shoot darts with a hypodermic needle tip, filled with a dose of tranquilizer solution that is either sedative or paralytic, which once injected will temporarily impair the target's physical function to a level that allows it to be approached and handled in a resistless and thus safe manner. Figure 9 indicates the tranquilizer gun which is used to target the animal and shot it.

Tranquilizer guns have a long history of use to stun wildlife when they are in a place where they pose a threat to others and themselves without having to kill the animal, or used to capture wildlife risking serious injuries to both the hunter and the target. They are also used for recreation, which animal rights groups protest against. Tranquilizer darts can also be fired by crossbow or breath-powered blowgun.

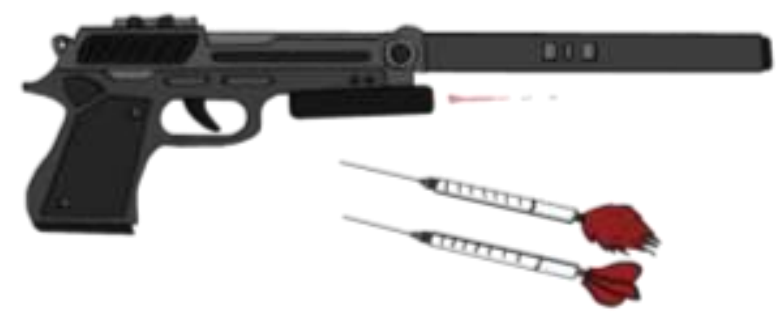

Figure 9 Tranquilizer Gun

\footnotetext{
$>\quad$ Advantages

1) It protects cultivable land from animals.

2) It reduces the effort of man power.

3) It provides safety to human and also animals.

4) Wireless control using VNC
}

\section{$>\quad$ Applications}

1) Wildlife safety measurement.

2) Agricultural field safety.

3) Human protection system.

4) Roadside safety system in forest area.

\section{CONCLUSION}

Use of image signal processing for tracking of wild animals makes it harmless and much more effective than the conservative methods like Electric fences, RFID and LF tags. Also, the future scope of this project can be extended to various purposes like roadside safety of travellers from wild animals in forest boundroads. 


\section{FUTURESCOPE}

This particular paper is limited to identifying the wild animals using image signal processing and broadcasting the same information to forest officers and villagers. But the consequent actions like sedating the wild animal would need human intervention. In future along with identification of wild animals, control measures like sedating based on animal type can be implemented with more real time data and advanced sensors and systems which would make this technique completely automated without any manual interference.

\section{REFERENCES}

[1]. S. Santhiya, Y. Dhamodharan, N E. KaviPriya, C S. Santhose and M. Surekha, "A smart farmland using Raspberry Pi crop prevention and animal intrusion detection system," International Research Journal of Engineering and Technology (IRJET), Volume:05, Issue:03, March- 2018, page:3829-3832.

[2]. Aryuanto Soetedjo, Ali Mahmudi, M. Ibrahim Ashari and Yusuf Ismail Nakhoda, "Raspberry Pi Based Laser Spot Detection," IEEE International Conference on Electrical Engineering and Computer Science, November 24-25, 2014, Bali, Indonesia, page:07-11

[3]. Divya, UshaKiran, and M. Praveen "IOT-Based Wild Animal Intrusion Detection System," International Journal on Recent and Innovation Trends in Computing and Communication (IJRITCC), Volume: 06, Issue: 07, ISSN:2321-8169, July 2018, pp:06-08.

[4]. S. J. Sugumar and R. Jayaparvathy, "An early warning system for elephant intrusion along the forest border areas," Current Science, Vol.104, pp.1515-1526, 2013. View at GoogleScholar.

[5]. S. R. Chourey, P. A. Amale et al, IETE Zonal Seminar "Recent Trends in Engineering \& Technology"-2017 Special Issue of International Journal of Electronics, Communication \& Soft Computing Science and Engineering, ISSN: 2277-947.

[6]. Son Kuswadi, Muhammad Nasyir Tamara, and Dwi Nugroho H W, "Gun Turret Automatic Weapon Control System Design and Realization". Published in: 2016 International Symposium on Electronics and Smart Devices (ISESD)

[7]. Sheela.S, Shivaram. K., Chaitra. U, Kshama. P, Sneha. K, Supriya. K" Low Cost Alert System for Monitoring the Wildlife from Entering the Human Populated Areas Using IOT Devices" International Journal of Innovative Research in Science, Engineering and Technology. (10 May, 2016) Vol. 5, Special Issue 10, May 2016.

[8]. Gokulraj, R. Krishnakumar, C. and Nanthakumar, M. "A Novel Approach of Intelligent Security System to Protect Farm Land from Wild Animals”. International Journal of Advanced Research Trends in Engineering and Technology. Vol. 4, Special Issue 11, March 2017. PP : 388 - 393, ISSN 2394-3777(Print), ISSN 2394-3785 (Online),

[9]. GophikaThanakumar, “An Automatic Detection System for Entry of Wild Animal”. International Journal on Recent and Innovation Trends in Computing and Communication ISSN: 2321-8169 Volume: 5, Issue: 5, PP: 1324 - 1326. 